\title{
Psicoterapia de Grupo para Mujeres con Embarazo de Alto Riesgo
}

\section{Group Psychotherapy for Women with High-Risk Pregnancies}

\author{
María Eugenia Gómez López Evangelina Aldana Calva Guillermo González Campillo \\ Cecilia Mota González Marco Antonio Sánchez Pichardo \\ Instituto Nacional de Perinatología \\ Isidro Espinosa de los Reyes, México
}

(Rec: 18 de mayo 2009 - Acep: 14 de octubre 2009)

\begin{abstract}
Resumen
El objetivo de este trabajo es presentar una propuesta de intervención psicoterapéutica con mujeres con embarazo de alto riesgo atendidas en una institución de salud reproductiva Ciudad de México. Por llevarse a cabo dentro de una institución, se plantea un modelo de intervención breve, focalizado, basado en el enfoque integrador de la psicoterapia. Se describen las principales características del grupo, la conflictiva presentada por estas mujeres, los temas abordados, así como las técnicas empleadas más relevantes.

Palabras clave: Psicoterapia de grupo breve, embarazo de alto riesgo, enfoque integrador.
\end{abstract}

\begin{abstract}
The aim of this work is to present a proposal for a psychotherapeutic intervention with women with high-risk pregnancies that are attending a reproductive health facility from Mexico City. Because it was carried out within a health institution, this is a model of brief intervention, focused, and based on the integrative approach to psychotherapy. It describes the main features of the group, the conflict presented by these women, the topics that emerged, and the most relevant techniques used.
\end{abstract}

Keywords: Brief group psychotherapy, high-risk pregnancy, integrative approach.

\section{Introducción}

Una de las líneas de la investigación psicológica mundial en las últimas décadas ha sido el estudio de la salud mental de la mujer durante el embarazo. Con base en los resultados obtenidos se propuso el concepto de salud mental perinatal, el cual incluye la vigilancia de los riesgos psicológicos que surgen en la mujer durante el periodo perinatal, y que pueden afectar tanto a la madre como al producto (Austin, 2003); e inducen a desarrollar estrategias de intervención para prevenir el desarrollo de psicopatología severa (Knop \& Osterberg-Larsen, 2001).

Durante el embarazo, las adaptaciones necesarias para el nacimiento de un nuevo ser afectan los ámbitos fisiológico, social y psicológico de la mujer, sin importar qué tan positiva sea su respuesta emocional frente al evento reproductivo. Esta es una etapa estresante que puede llegar a impactar posteriormente el vínculo con su hijo (Gómez \& Aldana, 2007).

Por lo común, frente a esta situación de ajuste y a los cambios de vida que implica un embarazo, la mujer manifiesta sintomatología depresiva o ansiosa que incide en su estado emocional y le genera malestar psicológico (Gómez, 2007).

Estas reacciones, que muchas veces son transitorias y adaptativas están supeditadas al evento reproductivo, debieran ser identificadas y atendidas a tiempo por parte del psicólogo, para evitar que se compliquen y se conviertan en un trastorno depresivo o ansioso que amerite otro tipo de tratamiento (Gómez, 2007; Gómez \& Aldana, 2007).

La literatura mundial muestra los resultados de diversos estudios acerca de la depresión y ansiedad perinatal (así como de la sintomatología depresiva y ansiosa), de su prevalencia, impacto y atención (Bernazzani, Saucier, David, \& Borgeat, 1997; Austin, 2004; Currid, 2004; National Collaborating Centre for Mental Health, 2006; Lara, Navarro, Navarrete, Cabrera, Almanza, Morales \& Juárez, 2006; Misri \& Kendrick, 2007; Orr, Blazer, James \& Reiter, 2007). Y aunque los resultados obtenidos varían de un país a otro, todos coinciden en concluir la necesidad de detectar $\mathrm{y}$ atender oportunamente estas alteraciones emocionales y evitar la aparición de trastornos mentales. 
Valorar de manera rutinaria la salud mental de la madre durante el embarazo y el posparto en un primer nivel de atención, tiene el potencial de identificar de manera temprana a aquellas mujeres que presentan malestar psicológico o factores de riesgo significativos para trastornos mentales, para evitar posteriormente el surgimiento de psicopatología (Austin, 2004; Austin \& Priest, 2005; Gómez 2007; Gómez \& Aldana, 2007; Gómez, Morales, Aldana \& Gaviño, 2008).

Esta valoración debe incluir la relación de la madre con el producto y con la pareja, el riesgo de daño a la madre y al infante, así como si existen pensamientos suicidas y problemas psicosociales como la exposición a la violencia doméstica (Austin \& Priest, 2005).

A pesar de que existen distintas posiciones acerca de la eficacia de la psicoterapia (Vera-Villarroel, 2004), los resultados de las revisiones sistemáticas realizadas sobre la psicoterapia durante el embarazo, plantean que dejar sin atender la enfermedad mental de la madre tiene consecuencias, no sólo para ella sino también para el feto en desarrollo, el infante, el niño o el adolescente. El trastorno mental de la madre, en particular en su forma más severa, se asocia también con deterioro significativo en el funcionamiento social y personal (National Collaborating Centre for Mental Health, 2006).

La psicoterapia durante el embarazo se recomienda para las alteración mental materna menos severa (Misri \& Kendrick, 2007), y deberá ser suficiente para estabilizar a la madre en trastornos depresivos y ansiosos de leves a moderados (Austin, 2004; Austin \& Priest, 2005). Para aquellas mujeres embarazadas que tienen un malestar psicológico que interfiere con su funcionamiento personal y social, pero que no reúne criterios diagnósticos para un trastorno mental, la consideración debe ser proporcionar apoyo social durante el periodo antenatal y posnatal. Éste puede ser de tipo individual o grupal informal pero regular (National Collaborating Centre for Mental Health, 2006).

Austin y Priest (2005) proponen que en la práctica clínica el tratamiento psicológico de la mujer embarazada necesita diseñarse de acuerdo con el tipo de trastorno, gravedad y el contexto psicosocial; determinando si habría que enfocarse en el estado de ánimo de la madre, la relación madre-hijo, las conductas de maternaje u otras variables.

En el Instituto Nacional de Perinatología "Isidro Espinosa de los Reyes", de Ciudad de México, institución pública de salud de tercer nivel donde se atienden los principales problemas de salud reproductiva, se cuenta con un Departamento de Psicología, el cual brinda, a través de diferentes programas de atención psicológica, apoyo psicoterapéutico a aquellas pacientes que lo requieren, uno de estos programas de atención es el dirigido a pacientes con embarazo de alto riesgo. A partir de la investigación realizada durante más de 20 años con esta población, se ha encontrado que sólo $4 \%$ de las pacientes con embarazo de alto riesgo presenta psicopatología severa, y entre 33.9\% y $62 \%$ manifiesta malestar psicológico como la alteración emocional más común durante este evento reproductivo (Luque, 1999; Gómez, Morales, Aldana \& Gaviño, 2008). Esto ha llevado a profundizar más en el estudio de dicha alteración emocional, lo cual se ha desarrollado ampliamente en trabajos previos (Espíndola, Morales, Díaz, Pimentel, Meza, Henales, Carreño \& Ibarra, 2006; Morales, Díaz, Espíndola, Henales, Meza, Mota \& Pimentel, 2004; Gómez \& Aldana, 2007; Gómez, Morales, Aldana \& Gaviño, 2008).

Con respecto al embarazo de alto riesgo, Gómez (2007) plantea que la alteración emocional por lo común es secundaria a factores reproductivos (embarazo, evolución del riesgo, pérdidas gestacionales previas, antecedentes de esterilidad, enfermedades crónicas anteriores al embarazo y temor a los procedimientos médicos y a la resolución del embarazo) y/o a factores psicosociales como disfunción marital, familiar y social.

Por lo anterior, el objetivo de este trabajo es proponer un modelo de psicoterapia institucional específico para esta población, para lo cual se hizo una revisión de la literatura sobre los tipos de psicoterapia con mujeres embarazadas y se tomó como base el modelo integrador.

La psicoterapia de grupo dentro de una institución de salud tiene como objetivo atender un número mayor de pacientes, y favorecer, a partir de la interacción de éstas, un proceso terapéutico que les permita, con base en las diferentes experiencias planteadas, alcanzar una conciencia de lo que las origina y, finalmente, encontrar maneras más adaptativas para solucionarlas.

El modelo de intervención que aquí se propone es un modelo integrador, ya que por las características de la población con la que trabajamos, así como por las necesidades de la institución, en nuestra práctica clínica nos hemos percatado que es necesario echar mano de diferentes técnicas provenientes de distintos enfoques teóricos para brindar el apoyo que la paciente requiere, es decir, debemos adaptar nuestras herramientas terapéuticas a las necesidades de nuestra población. Jiménez (2005) dice que anteriormente se hacía un esfuerzo por buscar pacientes adecuados para el método; sin embargo, las técnicas modificadas permiten un conjunto adaptativo de indicaciones, donde el tratamiento es el que se adapta a las características de cada paciente. Este planteamiento coincide también con lo propuesto por Norcross, uno de los teóricos del modelo integrador, quien afirma que un solo modelo de psicoterapia no puede ser adecuado para todos los casos. Este modelo surge a partir de la proliferación de diferentes psicoterapias y de la necesidad de conocer la efectividad de las mismas (Ruiz \& Cano, 2006).

El modelo integrador se compone de tres enfoques: 1) los factores comunes; 2) la integración teórica y 3) la integración técnica. 
El primero parte de la inducción lógica de que en las distintas psicoterapias hay una serie de procesos y factores comunes a todas ellas que explicarían este efecto general similar. Estos factores son: una relación de confianza; una explicación racional; proporcionar nueva información acerca de la causa y el tratamiento de los problemas del paciente; la esperanza del paciente de encontrar ayuda en el terapeuta; la oportunidad de tener experiencias de éxito y dominio de los problemas y facilitar la activación emocional.

Beyebach y Herrero de Vega (2007) profundizan en el estudio de estos factores comunes de las psicoterapias en contraposición a la especificidad de las mismas y exponen que la investigación de las técnicas terapéuticas y los factores comunes debe dirigirse a estudiar la interacción y sus efectos en situaciones clínicas reales y no tanto en contextos controlados y artificiales. También plantean que la atención en salud mental debe ser multidisciplinaria y el terapeuta debe ser capaz de intervenir con eficacia en una variedad de situaciones clínicas.

La integración teórica parte de la idea de que al unir dos o más enfoques de psicoterapia surgirá una mejor psicoterapia que las precedentes. Tratan de de integrar los elementos teóricos o conceptuales de las diversas psicoterapias, así como las técnicas que de ellas derivan (Ruiz \& Cano, 2006).

La integración técnica consiste en seleccionar procedimientos terapéuticos efectivos, independientemente de su marco teórico. Dicha selección puede darse con base en características de personalidad del paciente (Beutler) o de acuerdo a las dimensiones del funcionamiento humano (Lazarus), entre otras opciones (Ruiz \& Cano, 2006; Castelló, 2007). Sin embargo, existe otro enfoque al respecto propuesto por Calviño (1999), quien habla de una actuación psicoterapéutica más ajustada por sus límites y por su capacidad de impacto, y en un contexto institucional donde no existe mucho tiempo disponible para atender la demanda de los pacientes. En este sentido, este autor plantea la necesidad de tener un sistema más flexible y variado de recursos de intervención psicoterapéuticos que permitan al profesional no sólo una mayor capacidad de adecuación a las condiciones (de la situación, del paciente y de sî mismo), sino también una mayor capacidad de impacto productivo. Enfatiza y analiza que desde la práctica deben buscarse integraciones, cercanías, similitudes, relaciones de complementación; es decir, éste es un enfoque pragmático orientado a lo que es útil para el paciente en psicoterapia para lograr con ello un impacto terapéutico.

Con base en este enfoque, para este trabajo, consideramos que la psicoterapia para mujeres con embarazo de alto riesgo debe abarcar desde la consejería psicológica o counselling*, hasta, en algunos casos, intervenciones más

\footnotetext{
Tratamiento para problemas de aparición reciente (menos de un año), como son la adaptación a cambios en la vida, estrés y ansiedad situa-
}

profundas basadas en la psicoterapia psicoanalítica, de acuerdo con la problemática planteada por las mismas, así como con sus recursos emocionales para ahondar en sus conflictos y su apego al tratamiento psicoterapéutico.

Por lo anterior, a nivel teórico se toman como base los conceptos planteados por Bellak (1988) con respecto a la terapia breve, asimismo, se consideran algunos conceptos desarrollados por Fiorini (2000) en cuanto a la técnica de la psicoterapia focalizada y de Coderch (1987), con respecto a la psicoterapia psicoanalítica, a los conflictos básicos y derivados y las principales técnicas. No obstante, se han realizado algunas adaptaciones de estos conceptos para aplicarlos específicamente al trabajo terapéutico con la mujer embarazada.

Se retoma el concepto de Bellak (1988) acerca de la psicoterapia como un proceso simple que comprende desconocimiento, aprendizaje y reaprendizaje, el cual implica pocas sesiones de intervención, con metas específicas, que puede prevenir la aparición de alteraciones crónicas, para que la persona alcance un mejor nivel de ajuste general del que tenía antes. Este autor planteaba que el psicoterapeuta tiene la obligación de atender a una persona lo mejor que pueda, tomando en cuenta las limitaciones de sus motivaciones y su accesibilidad psicoterapéutica. La brevedad del tratamiento exige el máximo orden posible en cuanto a la precisión del pensamiento y la mayor economía en la intervención.

La psicoterapia breve se centra en el desarrollo continuo del paciente dentro de las distintas etapas de su ciclo vital, así como el análisis de los conflictos cuya aparición puede estar vinculada con la salud física, las relaciones interpersonales y con el ambiente. Las metas son modestas y requieren que el psicoterapeuta modere sus expectativas de cambio. En cuanto al paciente, debe tener la capacidad para establecer una alianza terapéutica en corto tiempo y para trabajar consigo mismo fuera de las sesiones; debe poseer una fuerza yoica mínima, una motivación adecuada y capacidad de respuesta a las interpretaciones (Ursano \& Hales, 1986, citado en Vives \& Lartigue, 1994).

Fiorini (2000) plantea que para cubrir las demandas de las instituciones de salud es necesario desarrollar psicoterapias breves que logren una comprensión de la vida cotidiana del paciente, que se instrumente en las interpretaciones, en la planificación de su vida diaria, en orientación familiar o laboral, para clarificar el interjuego entre el mundo interno y externo. Esta psicoterapia debe orientarse hacia el fortalecimiento de las "áreas del yo, libres de conflicto", para lograr, mediante el esclarecimiento de aspectos básicos de la situación del paciente, un fortalecimiento en su capacidad de adaptación realista, de discriminación y rectificación

cional, depresión subclínica o estado de ánimo disfórico, problemas maritales y relacionales, así como dificultades en las relaciones interpersonales (Bernard, Defey, Garbarino, Tutté \& Villalba, 2004). 
en grado variable de significaciones vividas. Con base en esto propone un modelo focalizado, que se orienta hacia la delimitación de un eje o punto nodal de la problemática del paciente, en el cual hay una interpretación central sobre la que se basa todo el tratamiento y el terapeuta debe llevar al paciente hacia la función y significado de sus síntomas. En este proceso intervienen factores reguladores desde el paciente, su familia, desde el terapeuta y la institución y desde el proceso terapéutico mismo que sistematizan y delimitan las situaciones a trabajar durante la terapia.

La iniciativa personal del terapeuta, la individualización, planificación, focalización y flexibilidad, son parámetros específicos de la psicoterapia breve que le dan estructura.

Con respecto a las técnicas utilizadas durante la psicoterapia, se retoman aquellas propuestas por Fiorini (2000).

Estas técnicas son: interrogar, informar, confirmar o rectificar, clarificar, recapitular, señalar, sugerir, confrontar; de las cuales, Coderch (1987) también toma en cuenta la clarificación y la confrontación.

Coderch (1987) plantea que la psicoterapia psicoanalítica, aunque se deriva del psicoanálisis, tiene su propio campo de acción, su técnica específica y sus objetivos propios, los cuales no son tan amplios y ambiciosos y están bien determinados, tienen características peculiares y su acción se dirige a desaparecer una sintomatología específica o a resolver una situación perturbadora del paciente, a través de trabajar con los conflictos derivados, para proporcionar al paciente una mayor disponibilidad de sus recursos mentales y atenuar, indirectamente, la patología intrapsíquica fundamental. Asimismo, considera al proceso terapéutico como una actividad de autoinvestigación que se dirige a articular, comprender y descubrir. Este autor también habla de foco en la psicoterapia psicoanalítica y lo define como aquellos síntomas y sufrimientos que aquejan al paciente y lo hacen buscar un tratamiento. La psicoterapia psicoanalítica permite una mejoría del sentido de realidad, y en su conjunto permite un enfrentamiento más positivo con el mundo circundante y las relaciones interpersonales.

Por conflicto básico, Coderch (1987) entiende la lucha que se da entre las pulsiones instintivas, la realidad externa y el superyó y que son mediadas por el yo a través de la formación de síntomas. Los conflictos derivados son perturbaciones producidas porque, con el paso del tiempo, los conflictos básicos van enraizándose en las circunstancias reales de la vida del paciente, dando lugar a alteraciones y disfunciones en éstas.

\section{Psicoterapia de grupo para mujeres con embarazo de alto riesgo}

Con base en lo anterior, se propone como objetivo principal de la psicoterapia de grupo para mujeres embarazadas con riesgo, la ampliación de la conciencia, el fortalecimiento de sus funciones yoicas y la posibilidad de afrontar de manera adaptativa los cambios en las situaciones de vida que implica el embarazo en sí mismo.

Por realizarse en una institución, es un modelo breve de intervención, que en el caso de las mujeres embarazadas se circunscribe a la duración de este evento.

La atención psicológica de la mujer embarazada deberá establecerse de acuerdo con los objetivos propios de cada institución; sin embargo, en general, nuestra propuesta implica aplicar un instrumento de tamiz como el Cuestionario General de Salud (CGS) a todas las pacientes que ingresen a la institución, para detectar a aquellas cuyo embarazo represente algún riesgo de tipo médico, que padezcan malestar psicológico y que esto pudiera afectar su adaptación a la maternidad y su apego al tratamiento médico dentro la institución.

Debe considerarse, además, que este modelo se elaboró para aplicarse en instituciones de salud cuyo objetivo principal no es la salud mental y emocional de las pacientes, sino que éste se circunscribe a la atención médica de alguna enfermedad o específicamente del embarazo, y por tanto, la atención psicológica es principalmente de apoyo para el tratamiento médico central que se le proporciona dentro de la misma. Por ello, la intervención del psicólogo en este tipo de instituciones puede verse limitada y supeditada al tratamiento médico, sobre todo tratándose del embarazo, donde en algunos casos la mujer debe mantener reposo prolongado, someterse a algún procedimiento médico previo, lo cual podría afectar su asistencia y su apego a un tratamiento psicológico.

\section{Criterios de inclusión al grupo}

Las pacientes embarazadas que ingresen a psicoterapia de grupo deben tener las siguientes características:

1. Estar embarazada y ser paciente de la institución que la atiende. El Servicio de Psicología, a partir de la historia clínica, establecerá el diagnóstico psicológico correspondiente, en el que se determina que la paciente presenta alguna alteración en su estado emocional a raíz de su embarazo. No importa en qué etapa del embarazo se encuentre, ya que siempre será posible incluirla en la dinámica del grupo.

2. Edad de 18 a 45 años. En este punto, la edad límite es relativa, pues en realidad no existe un tope establecido, todo depende de la capacidad reproductiva de la mujer. Sin embargo, para el manejo de la dinámica grupal sí es importante tomar en cuenta en qué etapa de su ciclo vital se encuentra, pues no es lo mismo una joven de 18 años que se embaraza sin planearlo ni desearlo y que por ello ve truncado de momento su proyecto de vida (estudiar y trabajar, por ejemplo); a una mujer entre los 25 y 35 años que desea este embarazo y se encuentra 
en el mejor momento de su etapa reproductiva, o a una mujer mayor, cuyo ciclo vital se orienta más a tener una vida más independiente pues ya tenía hijos adolescentes o adultos y que, sin desearlo, vuelve a embarazarse en una edad que implica muchos riesgos para ella y para el producto. No obstante, debe aclararse que no necesariamente todos los casos deben ser así, pero sí es importante que el clínico siempre considere la etapa del ciclo vital en que se da el embarazo, pues esto va a matizar la percepción que la mujer tenga de él y el impacto en su estado emocional.

3. Presentar alguna alteración emocional que pudiera afectar la evolución del embarazo.

Desde la historia clínica, el psicólogo establecerá el tipo de alteración emocional que presenta la paciente, si es un malestar psicológico supeditado al evento reproductivo, o si muestra sintomatología previa, exacerbada por el embarazo, que refiera a un proceso patológico anterior.

4. Pacientes psiquiátricas o neurológicas sólo con tratamiento médico. En estos casos es indispensable que la mujer embarazada reciba paralelamente el tratamiento farmacológico pertinente para su condición, no suspenda las consultas correspondientes y que el especialista informe periódicamente acerca de su evolución y pronóstico.

\section{Encuadre}

Las principales características por las que se rige el trabajo del grupo son:

- El grupo es abierto y su permanencia en el mismo está delimitada por la duración del embarazo, es decir, la paciente acude a terapia las semanas que le falten para la resolución del embarazo (aproximadamente entre 6 y 15 semanas).

- Las sesiones son semanales con una duración de 90 minutos.

- El tamaño del grupo oscila por lo común entre 6 y 15 pacientes.

- En el posparto se realiza una entrevista de seguimiento con la paciente para valorar su estado emocional y, de ser necesario, se le canaliza a otra institución para que continúe con su psicoterapia.

\section{Motivo de consulta}

Por lo general, la mujer embarazada que asiste a psicoterapia de grupo plantea de manera muy concreta que su estado emocional se ha alterado a raíz de su embarazo, manifestando principalmente sintomatología ansiosa o depresiva. Al momento de elaborar la historia clínica es posible precisar que el origen de dicha alteración, por lo común, tiene que ver con conflictos de pareja, familiares, o por problemas obstétricos previos, que son motivos de consulta manifiestos, los cuales entran en choque con conflictos que no son conscientes para la mujer y que están asociados directamente con el embarazo y que son: significado de la maternidad para la mujer, temor a la responsabilidad y al compromiso, representación simbólica del bebé y sus expectativas hacia éste, temor a la pérdida, entre otros, a los cuales se considera motivos de consulta subyacentes.

Estos conflictos tanto conscientes como inconscientes de la mujer embarazada son los temas eje (focos) que se trabajan a lo largo de las sesiones de manera guiada, con el fin de favorecer la ampliación de la conciencia y la elaboración de las situaciones de conflicto. A diferencia de lo planteado por Fiorini (2000), se ha observado que durante la dinámica grupal no sólo surge un tema eje, sino que pueden aparecer varios, los cuales, finalmente, se relacionan unos con otros como propios del embarazo, por esto se trabaja con ellos durante la terapia simultáneamente si es necesario.

Áreas de conflicto como temas eje (foco) de la terapia

En un estudio no publicado todavía, realizado con las pacientes del INPer con embarazo de alto riesgo atendidas por el Departamento de Psicología, se encontró que entre $60 \%$ y $70 \%$ de las pacientes solicitan atención psicológica por problemas de pareja, de $21.7 \%$ a $48 \%$ tienen problemas con la familia de origen, $21.3 \%$ a $39.7 \%$ presentan problemas obstétricos previos y entre $29.4 \%$ y $37.3 \%$ manifiestan como motivo de consulta sintomatología ansiosa o depresiva asociada al embarazo; por lo que se consideran las principales áreas de conflicto emocional que tienen las pacientes con embarazo de alto riesgo.

\section{Conflictos manifiestos}

- Conflictos de pareja. Como se mencionó anteriormente, el embarazo no sólo impacta el estado emocional de la mujer, sino también el de su pareja. Por ello, muchas de las preocupaciones y conflictos de las mujeres embarazadas giran alrededor de la relación con su pareja y del papel que juega este nuevo hijo en su dinámica establecida (Austin, 2004). Muchos de estos conflictos son previos al embarazo, y lo único que hace éste es exacerbarlos. Durante la terapia se ha observado que las mujeres tienden a elaborar la fantasía de que un hijo puede mejorar o incluso salvar la relación de pareja; sin embargo, en la mayoría de los casos esto no es así, lo que origina nuevos conflictos en la mujer al ver que esta expectativa no se cumple, y por tanto, esto dificulta su adaptación al evento reproductivo. Por otra parte, la mujer tiende a evadir o a negar el darse cuenta de la problemática de su relación y de que sus expectativas hacia la pareja son irreales y de que no existe un proyecto en común. También en estos casos se aprecian 
dificultades por parte de la pareja para comprometerse realmente en la relación y es la mujer la que insiste en sostener algo que al mismo tiempo la afecta enormemente y por lo cual se desgasta. Detrás de esto existe un gran temor de la mujer a quedarse sola, lo que no sólo responde a su dinámica interna sino también a la presión social. Asimismo, factores psicosociales como problemas económicos, de vivienda y poco apoyo familiar y social, entre otros, tienden a afectar también la relación de pareja ante el embarazo, por la necesidad de solventar la nueva situación. Obviamente conforme se desarrolla el proceso, se intenta que la mujer amplíe su conciencia con respecto al gasto emocional que le implica intentar sostener situaciones que no se resuelven y la alteran. Por ello, durante la terapia se busca que encuentre maneras más adaptativas de afrontar este conflicto, poner los límites necesarios para que no se desgaste emocionalmente y pueda centrar su atención en ella misma y en su hijo.

- Conflictos con la familia de origen y política. El papel de la familia extensa es muy importante para la mujer embarazada. Sin embargo, en algunos casos dicho apoyo no está presente y esto tiende a alterar su estado emocional. Esto se da sobre todo en aquellas situaciones en las que el embarazo no es bienvenido por no darse con una pareja estable y sin la solvencia económica necesaria. Esto implica mucha presión para la mujer embarazada, pues también contraviene a las expectativas familiares puestas en ella como seguir estudiando, trabajar, casarse, etc., lo cual le origina sentimientos de culpa. Asimismo, el tener que educar sola a un hijo le genera temores, que además se ven reforzados por el punto de vista social. Hay otros casos en los que el conflicto se da con la familia política, esto sobre todo cuando la pareja, o la mujer, conviven con alguno de ellos o cuando no existe apoyo de su parte por alguna circunstancia en particular. Lo cual altera su estado emocional, creándole mucho estrés. Por ello, a través de la terapia se busca que la paciente tome conciencia de la situación que está viviendo y cómo le está afectando, y aprenda a poner límites para desprenderse de su familia de origen y pueda establecer y consolidar su propia familia.

- Problemas obstétricos previos. Las características de los embarazos de riesgo atendidos en el INPer, implican un factor materno o fetal, que afectará de manera adversa el resultado de la gestación (INper, 1998). Se ha observado en la práctica clínica cotidiana que situaciones previas como pérdidas gestacionales recurrentes, abortos espontáneos, óbitos, tener hijos con defectos congénitos o discapacidades, amenazas de aborto en el embarazo actual o riesgo de presentar otros problemas, genera en las mujeres un malestar psicológico persistente caracterizado por angustia, temor a perder el producto y sintomatología ansiosa o depresiva (Gómez,
2007), que debe ser atendido para evitar que se agrave, brindando consejería psicológica y contención en un primer momento, para que posteriormente las mujeres puedan afrontar sus temores, y comprender y manejar las emociones asociadas a los eventos previos, con el fin de que puedan asumir la maternidad.

\section{Conflictos subyacentes}

- Significado de la maternidad para la mujer. La percepción del embarazo y la maternidad se asocia emocionalmente con la identidad que la mujer ha conformado desde su infancia, a partir de la relación con su madre y matizada por las expectativas sociales que ésta ha internalizado, haciéndolas propias. Durante el trabajo terapéutico se revisa con la mujer embarazada esta percepción del embarazo y la maternidad, así como la relación establecida con su madre y la representación simbólica de la maternidad, para que pueda identificar sus roles como mujer y madre. En este punto cabe resaltar que muchas veces para la mujer es difícil distinguir una identidad que no se asocie con sus papeles de hija, esposa y madre, puesto que desde pequeña se le ha reconocido como dependiente, primero, de los padres, luego del esposo, y finalmente de los hijos; por lo que durante la psicoterapia se intenta que identifique sus propias cualidades, motivaciones, expectativas y metas individuales, con el fin de que pueda adquirir cierta independencia emocional y fortaleza yoica para enfrentar sus problemas, sin necesidad de que nadie más lo haga por ella, y esto le permita asumir su rol de madre de manera más adaptativa.

- Temor a la responsabilidad y el compromiso. Uno de los más grandes temores que presenta la mujer embarazada es el de si está capacitada para hacerse cargo de su hijo, de cuidarlo y criarlo adecuadamente. En algunos casos, estos temores se manifiestan directamente a través de las fantasías que se tienen de cómo será la vida con su hijo; a partir de ello puede hacerse evidente si la mujer es capaz de anticipar esto o no, pues en otros casos existe una tendencia en ella a seguirse haciendo cargo de los problemas de los demás. Se observa también, una dificultad para dejar esta actitud y asumir un mayor cuidado hacia ella y su hijo, por temor a aceptar la responsabilidad y a comprometerse con su nuevo papel. Por ello, no es extraño encontrar que la mujer no se ha preparado para la llegada de su hijo, que no cuenta con ropa, ni la cuna, etc., arguyendo que aún le queda tiempo para ello, a pesar de lo avanzado de su embarazo. Ejemplos como éstos son indicadores de este tipo de temores y de cómo la mujer evade y posterga lo más posible asumir su maternidad cada vez más cercana. La terapia se focaliza en el reconocimiento del abandono de sí misma como rasgo biográfico y la consecuente dificultad para superarlo. 
- Representación simbólica del bebé y expectativas hacia éste. Como se mencionó antes, es muy importante que durante el proceso terapéutico se ayude a la mujer a reconocer qué espera del hijo que va a tener, pues a partir de ello podrá reconocerse el papel que éste juega en su psicodinamia. Es común encontrar que la mujer le da al bebé un valor importante como salvador de su relación de pareja, o como alguien con quien compensar lo que ella no tuvo, como una posesión, como quien confirma su fertilidad ante problemas previos de esterilidad, entre otros, y todo esto determinará el vínculo que establecerá con él. Asimismo, esto revive en ella, por un lado, las carencias infantiles del propio vínculo con su madre, y por otro, situaciones de conflicto más actuales como son su relación de pareja o su fertilidad. Otro tema que sale comúnmente durante la terapia es la preferencia por el sexo del bebé; esto en muchos casos también es motivo de conflicto, porque para algunas mujeres podría ser determinante para establecer un vínculo adecuado con él, ya que la remite a sus vivencias infantiles, las cuales en algunos casos fueron traumáticas: "que no sea niña para que no sufra lo que yo sufrí”. Con esto se ponen de manifiesto los mitos que existen alrededor de lo que es un niño o una niña.

- Temor a la pérdida. En ocasiones la mujer manifiesta temores, a veces reales, a veces no, de que pudiera pasarle algo a ella o a su bebé. Esto también incide en su estado emocional. En aquellos casos en los que la posibilidad es real por algún riesgo médico, durante la terapia se ayuda a la paciente a identificar lo que le está causando angustia y asimismo, se le ayuda a que anticipe cualquier situación que pudiera presentarse, con el fin de que sea capaz de afrontarla de mejor manera. En los casos en los que la posibilidad de que le suceda algo a ella o a su hijo es irreal y es generada por angustia, se intenta clarificar con ella los orígenes de sus temores, que muchas veces tienen que ver más con experiencias anteriores, y esto lleva a sobrevalorar al producto. Sin embargo, estos temores podrían haber estado desde antes del embarazo pero se manifestaban de otras formas, sobre todo si la mujer presentaba rasgos obsesivos y una gran ansiedad, que ahora se dirigen hacia el producto. En estos casos, se busca que la paciente identifique el origen del mismo y se intenta que tome conciencia acerca del papel que éste ha tenido en su dinámica interna.

\section{Papel del terapeuta}

El papel del terapeuta dentro del proceso terapéutico con mujeres embarazadas es muy importante para el desarrollo de la psicoterapia. Como tal, éste debe poseer una formación especializada como psicoterapeuta y debe tener además experiencia en el manejo de los grupos. Su papel dentro de la terapia breve es más orientador y de liderazgo, a diferencia de la psicoterapia más prolongada. De acuerdo con lo planteado por Bellak (1988) y Fiorini (2000), el terapeuta debe basarse en hipótesis de trabajo establecidas desde la elaboración de la historia clínica, para que, a partir de ellas, elabore un plan de trabajo específico y determine los temas eje (focos) a trabajar.

Según Bellak, el papel del terapeuta es más activo, pues debe explorar e interrogar hasta obtener la información necesaria por parte del paciente. Sus principales características son: a) empatía, b) calidez, c) espontaneidad, d) iniciativa y liderazgo, e) actitud docente, f) motivar hacia la tarea, g) clarificar los objetivos, h) reforzar todo avance en la tarea, i) claridad en su método expositivo.

Con respecto al tipo de intervenciones que el terapeuta tiene dentro del proceso terapéutico, Fiorini (2000) destaca lo siguiente:

a) Pedir y emitir información al paciente en forma de diálogo.

b) Plantear el encuadre estableciendo el tiempo del tratamiento, las condiciones del mismo, los derechos y obligaciones del paciente, así como su relación con la institución.

c) Intervenciones de esclarecimiento, señalamientos y confrontaciones, en las que se explicitan aspectos significativos de la conducta del paciente, dirigir su atención hacia los puntos clave de su comunicación, reformular los mensajes clarificándolos, y destacar las contradicciones entre lo verbalizado y la conducta actuada.

\section{Alianza terapéutica}

Valdivieso (1994) afirma que la calidad de la alianza terapéutica es de fundamental importancia en la producción de cambio a través de la psicoterapia y que alrededor del $40 \%$ de la variabilidad con respecto a la eficacia en las psicoterapias podría deberse a la intensidad y calidad de la alianza terapéutica.

En este mismo sentido, Krause, de la Parra, Arístegui, Dagnino, Tomicic, Valdés, Vilches, Echávarri, Ben Dov, Reyes, Altimir y Ramírez (2006) mencionan que dicha alianza se considera central en el proceso de cambio y se le atribuye la posibilidad de predecir los resultados de la terapia y ser responsable de gran parte de su efectividad. Asimismo, hablan de tres dimensiones de la alianza terapéutica que pueden tener un efecto positivo en el resultado de la terapia: el lazo personal entre terapeuta y paciente, el acuerdo de ambos con respecto a las metas y el acuerdo acerca de las tareas terapéuticas.

$\mathrm{Al}$ respecto, se ha observado en el trabajo con mujeres embarazadas que, a pesar de que en promedio asisten a alrededor de cinco sesiones de psicoterapia, la relación con el terapeuta influye para su apego al tratamiento y las motiva a generar cambios en su entorno que le ayuden a estar más tranquila. La empatía por parte del terapeuta, el establecimiento de metas de trabajo muy concretas, así como la 
clarificación de los problemas que presenta la paciente y la confrontación de los mismos, favorece la respuesta de cambio de las mujeres.

Con base en esto, el terapeuta que trabaja con mujeres embarazadas deberá establecer un encuadre como el mencionado anteriormente, que ante todo responda a las necesidades de la institución en pro de la paciente. Además deberá distinguir los temas eje a trabajar con base en el motivo de consulta: conflictos manifiestos y subyacentes propios de la dinámica de la mujer embarazada. Deberá tener siempre en mente los objetivos de este tipo de terapia, a saber: la ampliación de la conciencia, el fortalecimiento de las funciones yoicas y mejores estilos de afrontamiento para la solución de problemas, ya que su trabajo fundamental será que la mujer pueda adaptarse al evento de manera que no le implique un gasto emocional innecesario y le permita anticipar y afrontar sus circunstancias de vida con mayor fortaleza.

Por otra parte, el terapeuta que trabaja con mujeres embarazadas debe conocer ampliamente las manifestaciones propias de la personalidad de éstas, así como las fases por las que transcurre desde el punto de vista emocional, para que pueda orientar mejor sus intervenciones.

\section{Objetivos del trabajo psicoterapéutico}

Dada la limitación de tiempo y las necesidades institucionales, la psicoterapia de grupo con mujeres embarazadas se orienta a trabajar aspectos muy concretos que son comunes a éstas, principalmente:

1. Ampliación de la conciencia

2. Fortalecimiento de las funciones yoicas

3. Estilos de afrontamiento y solución de problemas

A continuación se explica cada uno con mayor detalle.

1. Ampliación de la conciencia. Cuando existe malestar psicológico, la conciencia se restringe temporalmente dado que la situación que lo origina rebasa la capacidad de respuesta del yo. Por ello, para la mujer embarazada es difícil encontrar soluciones adaptativas a los conflictos surgidos a partir de su embarazo. A través de la psicoterapia se busca que esta conciencia se amplíe en cuanto es posible restaurar las funciones yoicas que se inhibieron debido al impacto de la situación de conflicto.

2. Fortalecimiento de las funciones yoicas. Si se parte de que, en su mayoría, las mujeres presentan una disminución de sus funciones yoicas en respuesta al embarazo como una situación de ajuste, a través de la psicoterapia se intenta restablecerlas y fortalecerlas para que la mujer pueda irse adaptando a su embarazo, sin necesidad de que los demás intervengan o manejen la situación. En general, se busca que su capacidad de juicio, sus procesos del pensamiento, su sentido de realidad acerca del mundo y de sí misma, su capacidad de dominio y competencia, entre otras, le ayuden a ver su problemática desde otra perspectiva que al mismo tiempo le ayude a encontrar otras soluciones.

3. Estilos de afrontamiento y solución de problemas. Por lo general, las pacientes al inicio del embarazo tienden a negarlo o evadirlo como una manera de responder al impacto que esto les causa y, por tanto, como un estilo para afrontar la problemática. Sin embargo, esta actitud muchas veces se prolonga y la mujer no encuentra otra manera de enfrentar el embarazo, lo que le causa una alteración emocional que le impide encontrar soluciones adaptativas que le permitan asumir el control. En este sentido, durante la psicoterapia la paciente va narrando las situaciones que le causan conflicto y el terapeuta clarificando y señalando cómo ha sido que las cosas han llegado hasta ese punto, favoreciendo la relación entre los hechos y ampliando la conciencia con respecto a los mismos, para que las pacientes encuentren maneras más adaptativas de solucionar los problemas de forma independiente.

\section{Fases del grupo psicoterapéutico y manejo psicológico}

Por lo común, son pocas las pacientes que acuden a atención psicológica durante el primer trimestre de embarazo, en su mayoría inician su atención a partir del segundo trimestre. Esto se explica porque las mujeres a veces confirman su estado alrededor de las 7 u 8 semanas de gestación, y mientras ingresan a alguna institución médica para su atención e inician sus consultas, pasan aproximadamente 2 a 3 semanas. Sin embargo, para ilustrar la situación emocional durante el embarazo, se proponen tres fases del grupo que se asocian con los tres trimestres del embarazo y una fase de cierre de la experiencia.

Primer trimestre del embarazo. En esta fase son pocas las pacientes que ingresan al grupo psicoterapéutico, las que lo hacen están alrededor de la semana 10 a 12 de gestación. Sin embargo, es evidente en esta etapa que la mujer se encuentra en una situación de ajuste, en la que aún no asume que está embarazada, incluso le cuesta pensar en la posibilidad. Asimismo, entra en choque con lo que esto implica, pues cambia la visión de sus metas hasta ese momento para empezar a abrir un espacio emocional a un nuevo ser. Esto le genera temores y ansiedades, pues no sabe si podrá ser una buena madre y teme a lo desconocido por ser una experiencia nueva. Se ha observado que esta situación se incrementa tanto cuando este embarazo ha sido muy deseado y buscado, sobre todo cuando se han tenido problemas de esterilidad o existen pérdidas previas, así como cuando no lo es, por lo que predominan en la mujer ansiedad y temores por que este embarazo no se logre. 


\section{Técnicas}

Durante esta fase del trabajo terapéutico las técnicas se centran en la abreacción para facilitar la descarga emocional de los afectos asociados a sus temores y ansiedades acerca de la maternidad, y a partir de la intervención del terapeuta, primero se le brinda contención para tranquilizarla y ayudarla a comprender lo que le está sucediendo, y después se le clarifica y se le señala cómo esto se relaciona con otras vivencias para que amplíe su conciencia, con lo que se da inicio al proceso terapéutico como tal.

Segundo trimestre del embarazo. Durante esta fase la mujer embarazada va adaptándose poco a poco a su nueva situación, sin embargo, surgen en ella una serie de conflictos con el exterior en donde socialmente se le cuestiona la situación en la que se da su embarazo, si será capaz de ser una buena madre y si cuenta con una relación de pareja apropiada para ello. Por lo anterior, en esta fase los temas abordados por la mujer se orientan hacia la ansiedad que le causa su relación de pareja, si ésta es estable o no, si la pareja está entusiasmada con el embarazo o más bien lo rechaza, si existe la fantasía de que con este hijo salvará su relación, si su dependencia hacia la pareja es tal que no cree poder afrontar sola su maternidad. Al respecto, es en esta fase donde se hacen evidentes los verdaderos conflictos que la paciente tendrá que afrontar, el darse cuenta que su relación de pareja no funciona como ella creía, y que ésta depende más de lo que ella hace por conservarla que de lo que su pareja realmente es capaz de hacer por mejorarla, por lo que generalmente tiene que cerrar los ojos ante esta realidad con tal de seguir sosteniendo esta relación. Asimismo, se hacen evidentes los problemas de comunicación y las diferencias que existen en las expectativas que cada uno tiene hacia el embarazo y, por ende, hacia su papel como padres. Darse cuenta de esto genera mucha ansiedad en la mujer embarazada y ésta se incrementa por la presión que recibe de la familia en cuanto a la expectativa social que se tiene de ella y que no está cumpliendo.

Esto mismo se aplica en los casos en los que la mujer no tiene una pareja que asuma con ella su nueva responsabilidad, el conflicto es el mismo, es decir, éste se da entre la realidad que está viviendo y la presión social por haber incumplido las expectativas sociales de la maternidad como el producto de una relación estable y formalizada. Como se mencionó anteriormente, por esto se le considera un tema eje en la psicoterapia de la mujer embarazada, porque la relación de pareja influye y pone en tela de juicio la aceptación social del evento, lo que afecta el estado de ánimo de la mujer durante el mismo.

A este respecto buena parte de las sesiones de terapia giran alrededor, primero, de darse cuenta cuál es la problemática real que vive la mujer con su pareja, para después aceptarla y entonces sí buscar soluciones más adaptativas que le permitan mejorar su relación o, en algunos casos, incluso terminarla.

Otro tema eje durante esta fase es la percepción de la maternidad por parte de la mujer. Este aspecto también se ve muy influido socialmente a partir de lo que es ser una "buena madre", lo cual se da primeramente a partir de la relación de la paciente con su propia madre. Aunque al respecto no es la intención de la psicoterapia revisar a profundidad esta relación temprana establecida entre la paciente y su madre, sí se traen al "aquí y ahora" del proceso terapéutico aquellas experiencias que la ayudaron a integrar su identidad de una manera, de tal modo que le permitan entender y darse cuenta de cómo la afectan en su vivencia de la maternidad e incluso en su relación de pareja. Al respecto, a través de la psicoterapia se busca fomentar la autonomía, el reconocimiento de cualidades y habilidades propias, que también forman parte de su identidad como mujer, así como el establecimiento de metas individuales, para que con ello eleve su autoestima y se sienta capaz de encontrar por sí misma soluciones más adaptativas a su situación.

\section{Técnicas}

En esta fase las técnicas que se utilizan se orientan a cuestionar la realidad que vive la paciente para señalarle y clarificarle de qué manera esto se relaciona con su situación actual y confrontarla con cómo además le afecta en su estado de ánimo para que, a partir de ello, logre ampliar su conciencia. Finalmente, se busca fortalecer su yo a partir de la elevación de su autoestima con respecto al reconocimiento de sus cualidades y habilidades individuales, lo que además fomenta en ella un sentido de autonomía y autosuficiencia y le permite plantearse metas.

Tercer trimestre del embarazo. En esta fase, dado que se acerca el momento del parto, la mujer embarazada concentra su atención en esto y aprovecha las sesiones terapéuticas para compartir de nuevo sus temores hacia éste, a la intervención de los médicos y a cualquier procedimiento quirúrgico inesperado. Por supuesto estos temores se extienden a la salud del bebé y a que nazca bien. En esta fase se hacen evidentes también las creencias con respecto al parto que otras mujeres les han transmitido, lo cual en algunos casos pueden incrementar sus temores y confundirlas. Asimismo, se le ayuda a asociar estos temores también con la adquisición de una nueva responsabilidad y se le motiva a expresar sus sentimientos al respecto. En esta fase también, y de acuerdo con lo trabajado en las sesiones anteriores, las pacientes están en posibilidad de manejar de manera más adaptativa sus áreas de conflicto, enfrentándose a su situación y poniendo límites, lo que al mismo tiempo eleva su autoestima. Finalmente, se les ayuda a que establezcan metas a corto y mediano plazo, sobre todo en lo referente a la crianza de su hijo y, en los casos que así lo requieren, a la solución de sus problemas económicos a través de algún 
trabajo que las ayude a adquirir independencia a este nivel para poder sacar adelante a su hijo.

\section{Técnicas}

En esta fase las principales técnicas utilizadas son la clarificación y el señalamiento, con la intención de que la mujer comprenda el origen de sus temores y además se le aclaran algunas dudas en cuanto al parto, con el fin de bajar un poco su ansiedad y tenga más información al respecto.

\section{Cierre de la experiencia}

Esta fase no puede darse en todas las pacientes, pues no todas saben cuándo nacerá su hijo. Con aquellas que son programadas para cesárea puede cerrarse su participación directamente en el grupo, para lo que se pide a la paciente que resuma lo que ha trabajado y aprendido durante el proceso terapéutico, alentándola además a que exprese cualquier preocupación o asunto pendiente que tenga en ese momento. Tanto el grupo como el terapeuta realimentan a la paciente, destacándole sus principales logros adquiridos durante el proceso.

En el caso de aquellas pacientes que no tienen su parto programado, conforme se acerca la fecha de éste se les estimula a expresar sus preocupaciones o asuntos pendientes, realimentándolas y reafirmándolas en sus logros. Sin embargo, hay algunos casos en los que, por prescripción médica, la paciente debe permanecer en reposo unas semanas antes del parto y ya no puede asistir al grupo, para lo cual se le visita en el hospital inmediatamente después del mismo para saber cómo se siente y para ayudarla en la expresión de sus sentimientos al respecto, si así fuera necesario.

No obstante, en el caso de todas las pacientes, sea cual sea su situación final, se les visita en el hospital después de su parto para verificar cómo se encuentran y si es necesaria alguna intervención más. A todas se les da una cita posterior al mismo para confirmar que todo sigue bien, y en el caso de aquellas que requieran proseguir su psicoterapia, se les canaliza a alguna institución de salud mental para continuar su tratamiento.

\section{Técnicas}

En esta última fase las técnicas utilizadas son el acompañamiento y la realimentación de lo logrado durante la psicoterapia. La intervención realizada durante la estancia hospitalaria es breve, en algunos casos catártica y de acompañamiento, y en muy pocos casos de intervención en crisis.

\section{Conclusiones}

El embarazo es el evento reproductivo más importante en la vida de la mujer. Es un momento crítico y de maduración que pone a prueba las fortalezas de personalidad de la mujer. Asimismo, impacta la dinámica de su relación de pareja, lo que puede originar conflictos en ella.

Es por esto que cada vez se le da mayor importancia a la salud mental de la mujer durante el embarazo y a que lo viva sin alteraciones emocionales que pudieran repercutir en el desarrollo y culminación del mismo.

En las instituciones de salud, como parte de la atención integral que se brinda a la mujer con embarazo de riesgo, debería contarse con un programa para atender la salud psicológica de la mujer embarazada a través del Servicio de Psicología correspondiente. Por medio de éste puede proporcionársele la atención psicológica más adecuada en diferentes niveles de intervención. La psicoterapia de grupo es uno de ellos, la cual ha probado ser efectiva por la posibilidad de atender a un mayor número de pacientes en un periodo breve, con metas de trabajo muy concretas y con un modelo dividido en cuatro fases, de acuerdo con la respuesta psicológica de la mujer durante el embarazo, la cual va cambiando de un trimestre a otro.

Este modelo de intervención tiene como objetivos específicos favorecer el fortalecimiento del yo, la ampliación de la conciencia y la adquisición de estilos más adaptativos de afrontamiento y de solución de problemas, de tal modo que la mujer aproveche las semanas que le faltan para su parto para adquirir mayor conciencia de su situación, afrontarla de mejor manera y, asimismo, pueda desempeñar su papel de madre sin tantos conflictos que la pongan en riesgo de padecer trastornos mentales mayores y que pudieran afectar el vínculo con su hijo y su crianza.

\section{Referencias}

Austin, M-P. (2003). Perinatal mental health: opportunities and challenges for psychiatry. Australasian Psychiatry, 11(4), 399-403.

Austin, M-P. (2004). Antenatal screening and early intervention for "perinatal" distress, depression and anxiety: where to from here? Archives of women's Mental Health, 7, 1-6.

Austin, M-P. \& Priest, S. R. (2005). Clinical issues in perinatal mental health: new developments in the detection and treatment of perinatal mood and anxiety disorders. Acta Psychiatrica Scandinavica, 112, 97-104.

Bellak, L. \& Small, L. (1988). Psicoterapia breve y de emergencia (2a Ed.). México D. F.: Pax.

Bernardi, R., Defey, D., Garbarino, A., Tutté, J. C., \& Villalba, L. (2004). Guía clínica para la psicoterapia. Revista Psiquiátrica de Uruguay, 68, 99-146.

Bernazzani, O., Saucier, J-F., David, H. \& Borgeat, F. (1997). Psychosocial factors related to emotional disturbances during pregnancy. Journal of Psychosomatic Research, 42(4), 391-402.

Beyebach, M. \& Herrero de Vega, M. (2007). Factores comunes y procedimientos específicos en psicoterapia:; un divorcio "empíricamente validado"? (En red.) Disponible en: http//www3.usal.es/ master_psipia/ppersonales/art_mbb/6.PDF

Calviño, M. (1999). Premuras y corduras en psicoterapia. Sincronía, otoño, 1999. (En red.) Disponible en: http://fuentes.csh.udg.mx/CUCSH/ Sincronia/calvino.htm

Castelló, B. J. (noviembre, 2006). Integración y cambio en psicoterapia. (En red.) Disponible en: www.terra.es/personal2/jorge.consulta 
Coderch, J. (1987). Teoría y técnica de la psicoterapia psicoanalítica. Barcelona, España: Herder.

Currid, T. (2004). Improving perinatal mental health care. Nursing Standard, 19(3), 40-43.

Espíndola, J. G., Morales, C. F., Díaz, F. E., Pimentel, N. D., Meza, P., Henales, A. C., Carreño, M. J. \& Ibarra, A. C. (2006). Malestar psicológico: algunas manifestaciones clínicas en la paciente gineco-obstétrica hospitalizada. Perinatología y Reproducción Humana, 20, 112-122.

Fiorini, H. J. (2000). Teoría y técnica de psicoterapias. Buenos Aires, Argentina: Nueva Visión.

Gómez, L. M. E. (2007). Guía de intervención psicológica para pacientes con embarazo de alto riesgo. Perinatología y Reproducción Humana, 21, 111-121.

Gómez L. M. E. \& Aldana, C. E. (2007). Alteraciones psicológicas de la mujer con embarazo de alto riesgo. Psicología y Salud, 17(1), 53-61.

Gómez L. M. E., Morales, C. F., Aldana, C. A. \& Gaviño, G. F. (2008). Tipo de resolución del embarazo y estado emocional de la mujer. $\mathrm{Gi}$ necología y Obstetricia de México, 76(7), 365-372.

Instituto Nacional de Perinatología (1998). Normas y procedimientos de obstetricia y ginecología, México, D. F.: INPer.

Jiménez, J. P. (2005). El vínculo, las intervenciones técnicas y el cambio terapéutico en terapia psicoanalítica. Aperturas psicoanalíticas, 20, $1-19$.

Knop, J \& Osterberg-Larsen, B. (2001). Psychological intervention during pregnancy. A multidisciplinary hospital network. Ugeskrift for Laeger, 163(37), 5018-5022.

Krause, M., de la Parra, G., Arístegui, R., Dagnino, P., Tomicic, A., Valdés, N., Vilches, O., Echávarri, O., Ben Dov, P., Reyes, L., Altimir, C. \& Ramírez, I. (2006). Indicadores genéricos de cambio en el proceso psicoterapéutico. Revista Latinoamericana de Psicología, 38(2), 299-325.

Lara, M. A., Navarro, C., Navarrete, L., Cabrera, A., Almanza, J., Morales, F. y Juárez, F. (2006). Síntomas depresivos en el embarazo y factores asociados en pacientes en tres instituciones de salud de la Ciudad de México. Salud Mental, 29(4), 55-62.

Luque, C. M. M. H. (1999). Alteraciones emocionales asociadas con complicaciones ginecoobstétricas. Propuesta de un modelo de evaluación y de un programa preventivo. Tesis de Doctorado. Facultad de Psicología, Universidad Nacional Autónoma de México, México, D. F.

Misri, S. \& Kendrick, K. (2007). Treatment of perinatal mood and anxiety disorders. Canadian Journal of Psychiatry, 52, 489-498.

Morales C., F., Díaz F., E., Espíndola, H., J. G., Henales A., C., Meza R., M. P., Mota G., C. \& Pimentel N., D. (2004). Concepto de salud psicológica. En Universidad Iberoamericana (Ed.): Temas selectos en orientación psicológica. Creando alternativas (pp. 49-61). México: El Manual Moderno.

National Collaborating Centre for Mental Health (2006). Antenatal and postnatal mental health. Clinical management and service guidance. United Kingdom: National Institute for Health \& Clinical Excellence.

Orr, S. T., Blazer, D. G., James, S. A. \& Reiter, J. P. (2007). Depressive symptoms and indicators of maternal health status during pregnancy. Journal of Women's Health, 16(4), 535-542.

Ruiz, S. J. J. \& Cano, S. J. J. Psicoterapia por la personalidad (En red) Disponible en: www.psicologia-online.com/ESMUbeda/libros/Personalidad/personalidad.htm, (consultado en noviembre 2006).

Valdivieso, F. S. (1994). Fundamentos y límites de la psicoterapia. Boletín de la Escuela de Medicina de la Pontificia Universidad Católica de Chile, 23, 97-102.

Vera-Villarroel, P. E. (2004). Estrategias de intervención en psicología clínica: las intervenciones apoyadas en la evidencia. Liberabit, 10, 4-10.

Vives, J. \& Lartigue, T. Manual de psicoterapia breve durante el embarazo y la lactancia. México, D.F.: Universidad Iberoamericana, p. 28. 
